\title{
Paintball Thrombus in the Left Atrium Complicating Severe Mitral Stenosis
}

\author{
Jihad Raoui $^{{ }^{*}, \text { Elizabeth O.Ugoani }^{3}, \text { Bouthayna Mesmoudi }^{2} \text {, Nesma Bendagha }}{ }^{4}$, Rokya Fellat ${ }^{5}$ \\ ${ }^{1,2,3}$ Resident doctor Cardiology A Department, IbnSina Teaching Hospital Rabat \\ ${ }^{4}$ Cardiologist, Cardiology A Department, Ibn Sina Teaching Hospital Rabat \\ ${ }^{5}$ Professor, Assistant Head of Department Cardiology, IbnSina Teaching Hospital Rabat
}

*Corresponding Author: Jihad Raoui, Resident doctor Cardiology A Department, IbnSina Teaching Hospital Rabat, E-mail: raoui.jihad@gmail.com

\begin{abstract}
Left atrial floating thrombus is rare but it can be responsible for fatal embolic complications. It usually happens in a very dilated left atrium with a stagnation of the blood flow due to a severe rheumatic mitral stenosis with atrial fibrillation. We report a case of a floating thrombus of the left atrium in a mitral stenosis. Our patient is 68 years old, presented with a class II shortness of breath and palpitations, whose clinical examination found a diastolic bearingand a tricuspid regurgitation murmur.

The transthoracic echocardiography revealed a tight mitral stenosis with a floating thrombus in the left atrium, which seems to be totally free in the left atrium's chamber, and an important tricuspid regurgitation.

The patient was referred urgently to the cardio vascular surgery department where he had a mitral valve replacement with a thrombectomy, and a tricuspid plasty, with a positive evolution. The anatomopathological study confirmed the thrombotic nature of the left atrium floating mass.
\end{abstract}

Keywords: Paintball thrombus, left atrium, mitral stenosis

\section{INTRODUCTION}

The mitral stenosis is a valvular diseasethat is very frequent in the developing countries. The left atrium thrombus ball is a none pedicled free floating thrombus. Unlike the mural thrombus, it is a very rare complication of the tight mitral stenosis. The mitral stenosis and the dilatation of the left atrium drive to an important blood stasis, leading to the constitution of the thrombus.

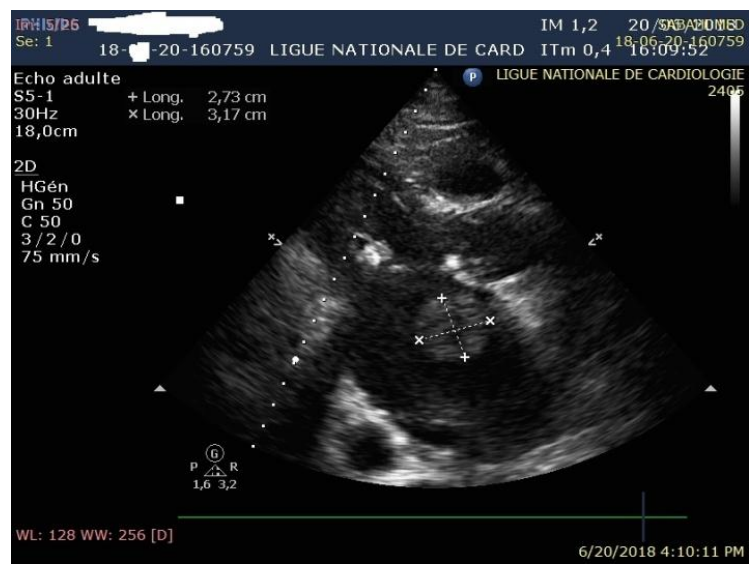

Fig1

\section{CASe Report}

A 68-year-old man presented with class II shortness of breath and palpitations, whose clinical examination found a diastolic bearing and a tricuspid regurgitation murmur.

The transthoracic echocardiography showed a tight mitral stenosis with a floating thrombus in the left atrium, which seems to be totally free in the left atrium's chamber, and an important tricuspid regurgitation. (Figure 1 and 2)

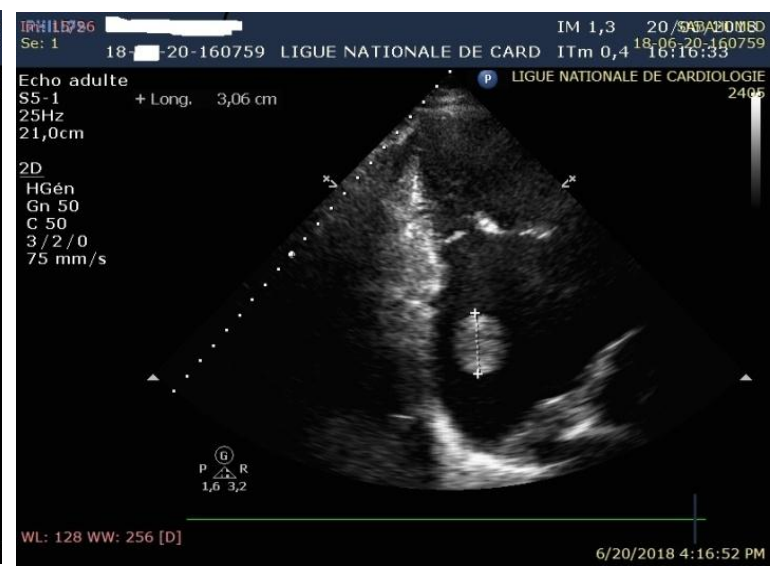

Fig2 
Figure1 and2. Echocardiographic images showing a floating thrombus in the left atrium measuring $30 \mathrm{~mm}$

The patient was referred urgently to the cardio vascular surgery department where he had a mitral valve replacement with a thrombectomy, and tricuspid plasty, with a positive evolution.
The anatomopathological study confirmed the thrombotic natureof the left atrium floating mass. (Figure 3)

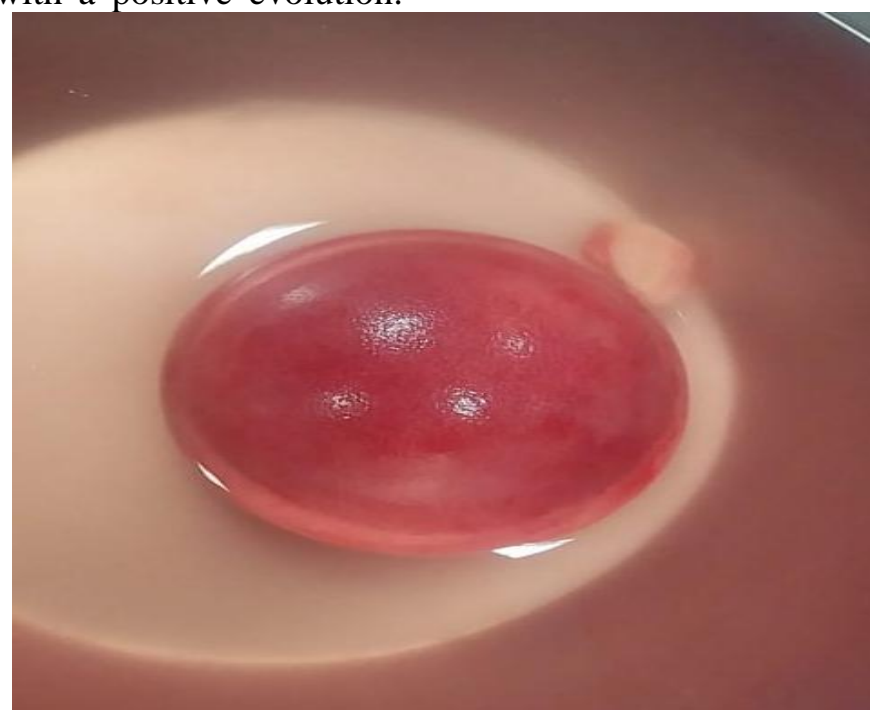

Figure3. Image showing the thrombus after a thrombectomy

\section{DISCUSSION}

The floating thrombus of the left atrium was first described by Wooden in 1814 with the autopsy of a young 15 year-old girl presenting a mitral stenosis. It is a rare entity that occurs mostly in the left atrium, in patients who have a mitral stenosis, especially when they have an atrial fibrillation.

Nowadays, thanks to the echocardiography, the CT and MRI, the diagnosis can be made earlier.

Some situations such as atrial fibrillation, mitral stenosis, left atrium dilatation, left ventricular severe dysfunction, and mitral valve prothesis, promote the constitution of a thrombus, and the risk of systemic embolism. Turhan described a floating thrombus in the left atrium in a 11 yearold boy, complicated of peripheral arterial embolism in the left popliteal artery in a restrictive cardiomyopathy with a sinus rhythm (4).

The free or floating thrombus is part of a subgroup of particular thrombi which are characterized by a special evolution and clinical consequences, dominated by the risk of orifical stuckand a particular macroscopic appearance combining a spherical shape with a smooth structure, which can be confused with a myxoma, without any septal or valvular attachment.

Abe and coll correlated the risk of systemic embolism in the atrial fibrillation to the shape, the localization, the mobility, the number, and the size of left atrial thrombi (1). Thus, the mobility of the thrombus seems to be a predictive factor of cerebral and peripheral embolic events, independently of the other factors.

\subsection{Etiopathogenesis}

The etiopathogenesis seems to be secondary to a mural micro-thrombus, which grows progressively, forming a mass projecting in the left atrium, and hanging to one of the walls with a pedicle. Then, the thrombus is going to become detached and thus, completely free, and get some morphological transformation (smooth surface) (3).

The floating thrombus usually starts from the atrium septum, in the form of a mural small thrombus, and less often from the left auricle. The constitution of the floating thrombus can result from an initial micro thrombus sticking to the wall, with an accretion phenomenon, which makes progressively increase the mass («snowball » effect). It becomes prominent but stays stuck to the atrial wall with a pedicle. The more the body become bigger, the more the pedicle gets thinner until the detachment or the fragmentation of the thrombus. While it is freely floating in the left atrium chamber, taken from an atrium wall to another, and having a rotary movement around its own axis, the thrombus gets secondarily its smooth and polished characteristic appearance. Strictly speaking, a 
free thrombus (free ball thrombus) has a smooth surface without any left attachment to one of the atrium walls, and a size which is usually bigger than the mitral valve.

In our case, it is a free-floating thrombus in the left atrium, which is very distended, with a tight mitral stenosis that seems to be rheumatic.

\subsection{Diagnosis}

A floating thrombus in the left atrium should be suspected in a patient who has a tight mitral stenosis with atrial fibrillation, and anintermittent or variable diastolic bearing, or who present embolic events or syncopes (2). Adiastolic bearing can increase when the free thrombus is partially stuck in the mitral valve, and on the contrary,temporarily disappear when the valve is obstructed. The symptoms variability is also associated to postural change. Indeed, the sitting position can exacerbate the dyspnea.

Grandmougin and coll. reported the case of a patient who had a giant left atrial floating thrombus, in the absence of mitral stenosis, with a paroxysmal postural dyspnea as a single symptom. Sometimes, the floating left thrombus can stay asymptomatic for a long time. Vitale and coll. reported the case of a patient who has a left atrial floating thrombus associated to a tight mitral stenosis, without any clinical signs or complications due to this situation, despite the huge size of the thrombus.

In our case, the patient's physical findings are very suggestive of the diagnostic and related to the temporary and partial stuck of the thrombus in the mitral valve.

In general, the echocardiography certifies the diagnosis when a floating thrombus is clinically suspected, and should be done without further delay.

The bidimensional transthoracic echocardiography is the non-invasive method of choice for detecting cardiac masses.

\subsection{Risks Due to a Floating Thrombus}

During the development and the morphological changes of the thrombus, the patient can present temporary embolic complications or cerebrovascular ischemic strokes. In fact, the fragmentation of the thrombus followed by a peripheral embolism can cause ischemia or infarction in the myocardium, the brain, the guts, or the extremities.
Paradoxically in the literature, the systemic emboly is rarely reported in the case of a free thrombus. It seems to be related to the endo the lialization of the surface of the thrombus which integrity prevent from platelet aggregation and fragmentation.

In our case, the severity of the mitral stenosis probably disabled the thrombus crossing, even if it was totally free.

However, the association of the floating thrombus to embolic events was described in the literature and results either from the fragmentation of the free thrombus which is projected against the mitrale valve in each ventricular systole, or from factors which are independentfrom the thrombus, and related to the left atrium ( medical history of mitrale surgery,chronic atrial fibrillation ).

Furthermore, the free movements of the thrombus could cause syncope, an acute pulmonary oedema, or a sudden death according to the degree of stenosis of the mitrale valve (occasional, incomplete, or complete respectively).

Thus, the first step of a micro-thrombus is dominated by the risk of embolism whereas the main consequence of a floating thrombus is an acute hemodynamic decompensation and the risk of a sudden death due to an obstruction of the mitrale valve («hole-in-one» thrombus effect).

Sometimes, the embolic and obstructive phenomenon can occur at the same time.In the case of aventricular floating thrombus, the risk of sudden death increases. («hole-in-two» thrombus effect).

\subsection{Urgent Surgery}

The discovery of a free thrombus in the left atrium should give rise to the indication for urgent surgery to avoid fatal complications, especially when some of these echocardiography criteria are found: surgical mitral stenosis, dilated left atrium, complete arrhythmia with atrial fibrillation, complete mobility, size of the mass > $1 \mathrm{~cm}$, multiple floating thrombi, associated ventricular localization(«hole-in-two thrombi » effect).

The surgery enables a survival rate that is superior to90 \%. After the thrombectomy, some additional procedures should be considered in the managing of a free left atrial thrombus, according to the preoperative information. Thus, the reducing of the left atrium, a mitral valve 
replacement, the ablation of the atrial fibrillation with radiofrequency or a Cox Maze procedure should be considered. (7)

The fibrinolytic and the anticoagulant treatment should not be considered in the immediate free thrombus managing. However, some cases of floating thrombi disappearing with anticoagulants have been reported in the literature, but this requires a very close echocardiographic monitoring and an extension of the length of stay until the complete disappearing of the mass. Whatever be the case, the unpredictability of the evolution of the floating thrombus and the hemodynamic major risk of this disease inflict a surgical treatment as soon as possible, which is the case of our patient.

\section{CONClusion}

The left atrial thrombus is a classic complication of mitral stenosis, especially if there is an atrial fibrillation and an atrial distension.It is associated to serious complications such as an obstruction of the mitral valve and a sudden death, or the fragmentation and peripheral embolization. The medical treatment by itself with anticoagulants or thrombolytic is reserved for the very serious cases, which forbid the surgery, or for patients who reject the intervention.

\section{REFERENCES}

[1] F Nya, N Atmani, A Seghrouchni, A Abdou, S Bellouize, Y Moutakiallah, M Bamous, M Drissi, GA Ghadbane, M Aithoussa, Y Elbekkali, A Boulahya Service de chirurgie cardiaque, Hôpital militaire d'instruction Mohamed V, Faculté de médecine et de pharmacie, Université Mohamed V, Rabat, Maroc Thrombus flottant de l'oreillette gauche révélé par des embolies multiples

[2] N. Haddour, R. Mesbahi, M. Kettani, L. Chami, L. Amar, M. Kouach, M. Benomar Et M. Benomar, Chu De Rabat, Maroc Retrecissement Mitral Syncopal Lié À Un Thrombus Flottant

[3] Yeoh J, Yan C, Soo C, Lim Y, Choo H. Mitral stenosis and free-floating left atrial thrombus: demonstration of the "hole-in-one" effect by color Doppler echocardiography. Am Heart J. 1991; 121:1551-2 pubmed

[4] Turhan H, Ocal A, Erbay A, Yasar A, Cicekcioglu F, Yetkin E. Free-floating left atrial ball thrombus developed in an 11-yearold child with restrictive cardiomyopathy during sinus rhythm: manifested as a major thromboembolic event. Int J Cardiol. 2005;103 :111-3 pubmed

[5] Rider O, Malhotra A, Newton J. Free floating left atrial ball thrombus: a rare cause of stroke. J Stroke Cerebrovasc Dis. 2013; 22:e238-9pu bmedpublisher

[6] Lie J, Entman M. "Hole-in-one" sudden death: mitral stenosis and left atrial ball thrombus. Am Heart J. 1976;91:798-804 pubmed

[7] Nishimura R, Otto C, Bonow R, Carabello B, Erwin J, O'Gara P, et al. 2014 AHA/ACC guideline for the management of patients with valvular heart disease: a report of the American College of Cardiology/American Heart Association Task Force on Practice Guidelines. J Thorac Cardiovasc Surg. 2014;148:e1-e132 